\title{
Avaliação prognóstica de idosos em doenças crônicas e síndrome metabólica - fatores de risco para doenças cardiovasculares
}

\section{Prognostic evaluation of elderly people in chronic diseases and metabolic syndrome - risk factors for cardiovascular diseases}

DOI: $10.46814 / 1 \mathrm{ajdv3n2-004}$

Recebimento dos originais: 23/12/2020

Aceitação para publicação: 26/02/2021

\author{
Sonia Maciel da Rosa \\ Doutora em Ciências Biológicas pelo Instituto Nacional de Pesquisas da Amazônia. \\ Instituição: Universidade do Estado do Amazonas. \\ Endereço: Av. Djalma Batista, 2470 - Chapada, Manaus-AM. CEP: 69050-010. \\ E-mail: sonia.maciel.rosa@gmail.com
}

Janaína Batista de Freitas

Mestranda em Biotecnologia pela Universidade do Estado do Amazonas. Endereço: Av. Carvalho Leal, 1777 - Cachoeirinha, Manaus-AM. CEP: 69065-001.

E-mail: janainabatistadefreitas@gmail.com

\section{Sílvia Coelho Ribeiro}

Ciências Biológicas pela Universidade do Estado do Amazonas. Instituição: Universidade do Estado do Amazonas.

Endereço: Av. Djalma Batista, 2470 - Chapada, Manaus-AM. CEP: 69050-010.

E-mail: silvia.coelhoribeiro@gmail.com

\section{Rebeca Samantha Martelet David}

Estudante de Medicina pela Universidade Nilton Lins.

Instituição: Universidade Nilton Lins.

Endereço: Avenida Professor Nilton Lins, 3259 - Flores, Manaus, AM. CEP: 69058-030.

E-mail: rebeca_samantha@yahoo.com.br

\section{RESUMO}

As mudanças no perfil comportamental da população nas últimas décadas refletiram no estreitamento da pirâmide demográfica devido ao acelerado processo de envelhecimento da população, o que aliado a isso, é crescente o índice de Doenças Crônicas não Transmissíveis - DNCT's, em destaque, as doenças cardiovasculares nesse grupo. No Brasil são cerca de 17 milhões de portadores de hipertensão arterial, $35 \%$ da população de 40 anos e mais. A hipertensão é um dos mais importantes fatores de risco para o desenvolvimento de doenças cardiovasculares. É um grande desafio para o Sistema Único de Saúde - SUS, pois essas doenças que manifestam de forma silenciosa, são a grande causa de mortalidade nesse grupo. Com isso, o trabalho objetivou avaliar os fatores de riscos que contribuem para o surgimento de doenças cardiovasculares e assim apresentar soluções que ajudariam a reverter a situação em que se encontram. Para a realização do trabalho, contou-se com a participação de 123 voluntários, onde aos mesmos foi entregue um Termo sob o aval do comitê de ética, oferecendo-lhes informações esclarecedoras do trabalho. Posteriormente, foram submetidos a exames antropométricos; os dados foram discriminados e analisados conforme os padrões estabelecidos pela Organização Mundial da Saúde e Sociedade Brasileira de Diabetes. Os resultados obtidos foram que, em relação ao índice glicêmico, 6,5\% apresentavam-se diabéticos, 38\% hipertensos, 84,5\% com riscos para doenças 
cardiovasculares e 60\% acima do peso. Esses resultados foram apresentados aos mesmos em forma de palestras de retorno, apontando as dificuldades, fatores riscos que corroboram para o surgimento dessas doenças, e como podem ser controladas a fim de promover a melhoria na qualidade de vida desses idosos.

Palavras-Chave: DCNT's, população idosa, Doenças cardiovasculares.

\begin{abstract}
The chances in the behavioral profile of the population in the last decades reflected in the narrowing of the accelerated aging processo $f$ the population, wich allied to that, the index of chronic noncommunicable diseases - DNCT's, in highlight, cardiovascular diseases in this group. In Brazil there are about 17 million people with arterial hypertension, 35\% of the population aged 40 and over. Hypertension is one of the most important risk factors for the development of cardiovascular diseases. It is a great challenge for the Unified Health System - SUS, because these diseases that manifest silently, are the major cause of mortality in this group. Thus, the work aimed to assess the risk factors that contribute to the appearance of cardiovascular diseases and thus present solutions that would help to reverse the situation in which they find themselves. In order to carry out the work, 123 volunteers participated, where they were given a Term under the endorsement of the ethics committee, offering them clarifying information about the work. Subsequently, they underwent anthropometric examinations; the data were broken down and analyzed according to the standards established by the World Health Organization and the Brazilian Diabetes Society. The results obtained were that, in relation to the glycemic index, $6.5 \%$ were diabetic, $38 \%$ were hypertensive, $84.5 \%$ were at risk for cardiovascular diseases and $60 \%$ were overweight. These results were presented to them in the form of return lectures, pointing out the difficulties, risk factors that corroborate the emergence of these diseases, and how they can be controlled in order to promote an improvement in the quality of life of these elderly people.
\end{abstract}

Keywords: DCNT’s, elderly people, cardiovascular diseases.

\title{
1 INTRODUÇÃO
}

Mudanças na sociedade, relacionado a fatores tecnológicos, sociais e econômicos refletem, ao longo de décadas, no perfil comportamental da população brasileira, principalmente em relação ao aumento da expectativa de vida. Combinado com a diminuição do nível de fecundidade e os altos níveis de mortalidade, principalmente na população jovem, provocam modificações no padrão etário da população, que está envelhecendo rapidamente, contribuindo assim para o surgimento de Doenças Crônicas não Transmissíveis - DCNT's, gerando assim novas adequações para atender a demanda na área da saúde.

Para o Ministério da Saúde (2010), um ponto chave também a ser levado em consideração é a transição epidemiológica que caracterizam assim a mudança do perfil de morbimortalidade de uma população, ocorrendo progressivamente o aumento das doenças infecto-contagiosas contrapondo a elevação das mortes por doenças crônicas, aumentando o índice de mortalidade entre os idosos, conforme a tendência mundial. As doenças crônicas estão são doenças multifatoriais que se 
caracterizam por longo período de evolução e com períodos de remissão e exacerbação levando à incapacidade ou à morte (MANTOVANI et al., 2008).

No Brasil são cerca de 17 milhões de portadores de hipertensão arterial, 35\% da população de 40 anos e mais. A hipertensão é um dos mais importantes fatores de risco para o desenvolvimento de doenças cardiovasculares, cerebrovasculares e renais, sendo responsável por pelo menos $40 \%$ das mortes por acidente vascular cerebral, por $25 \%$ das mortes por doença arterial coronariana e, em combinação com o diabetes, $50 \%$ dos casos de insuficiência renal terminal (MINISTÉRIO DA SAÚDE, 2006).

Guyton (2011), salienta que a doença se dá pelo aumento da pressão arterial, acometendo uma a cada cinco pessoas antes do término da idade, sendo em geral na meia idade ou velhice. A doença pode provocar ruptura dos vasos sanguíneos cerebrais, bem como dos vasos renais causando a insuficiência renal, comprometendo órgãos vitais, produzindo a cegueira, surdez, ataques cardíacos, etc.

É hipertenso aquele indivíduo que possui a pressão arterial sistólica maior ou igual a $14 \mathrm{mmHg}$ e a diastólica, maior ou igual a 90mmHg, em indivíduos que não estão fazendo uso de medicação hipertensiva. Porém de acordo com os novos parâmetros discutidos no $8^{\circ}$ Joint National Committee (JNC 8) em 2013, existe um novo parâmetro para o estabelecimento da pressão arterial, observando um estudo sistemático de quadros clínicos, randomizados e controlados.

Wright Jr. et al., (2014) chegaram a discussão de novas metas para o estabelecimento de pressão arterial para o tratamento de hipertensão: são toleravéis para indivíduos hipertensos, abaixo dos 60 anos, a pressão até 140/90 mmHg, e 150/90 mmHg para indivíduos hipertensos acima dos 60 anos, com o intuito de diminuir o regime de medicamentos anti-hipertensivos. Porém, isso gera uma controvérsia pelo Departamento de Hipertensão da Sociedade Brasileira de Cardiologia, pois os mesmos alegam que, os famosos 120/80 mmHg continuam sendo a pressão ideal, com exceção dos hipertensos acima dos 60 anos, onde reduzir os níveis de pressão arterial com o tratamento um pouco acima dos níveis estabelecidos, é considerado satisfatório, o que é sumamente importante, levar em consideração o estudo do quadro clínico do paciente, analisando as suas particularidades.

A globalização, o consumismo, a necessidade de prazeres rápidos e respostas imediatas contribuem para o aparecimento da obesidade como uma questão social (MINISTÉRIO DA SAÚDE, 2006). O indivíduo que está adepto a esses hábitos, poderá também desenvolver a aterosclerose, que se dá pela deposição de gordura nos vasos sanguíneos, desencadeando um processo inflamatório que interromperá o fluxo sanguíneo provocando infarto do miocárdio, AVE - acidente vascular encefálico, doenças cardiovasculares e correlatas. 
A situação tende a se agravar, pois a falta de exercícios físicos e os maus hábitos alimentares ligados ao consumo de alimentos ricos em açúcar e gordura, como os fast-foods, alteram o metabolismo corporal, deixando-o mais lento, e consequentemente, pela deposição de tecido adiposo, o indivíduo é levado a condição de obeso.A obesidade é uma doença crônica, que pode ser classificada de acordo com o Índice de Massa Corporal ou Índice de Quetelet, utilizando a seguinte forma: IMC= Peso/(Altura $)^{2}$, onde, quanto maior for o IMC da pessoa, maiores serão os riscos de morte prematura e desenvolvimento de doenças crônicas. Para Nahás (1999), é considerado obeso o indivíduo que apresenta o IMC igual ou superior a 30, sendo um problema de saúde pública.

Existem várias consequências da obesidade: insuficiência cardíaca, diabetes, arteriosclerose, hipertensão cardíaca e aumento da mortalidade, assim um excesso de peso de $4.5 \mathrm{Kg}$ aumenta a porcentagem de mortalidade em torno de 8; um excesso de peso de $9 \mathrm{Kg}$ aumenta a porcentagem de mortalidade em 18 e assim por diante (LEDERER, 1991). Telema et al. (2007) explica que desde cedo, a obesidade pode ser estabelecida no indivíduo; onde Sidney et al. (1998) enfatiza que, a redução dos níveis de atividade física faz o indivíduo aumentar progressivamente, dos 20 aos 50 anos.

O diabetes também é uma das principais doenças crônicas que acometem a população idosa. A crescente prevalência e os altos níveis de morbimortalidade tornam o diabetes um grave problema de saúde pública nos países em desenvolvimento frente aos incrementos na expectativa de vida, uma vez que a prevalência da doença aumenta com a idade (PASSOS et al., 2005). Esta se encontra relacionada com a deficiência na produção de insulina devido a insuficiência pancreática, o que dificulta a entrada de glicose na célula, essencial para a síntese de energia, peça chave para o metabolismo celular e a homeostase da fisiologia humana. O diabetes é caracterizado por poliúria, polidipsia, perda de peso, acidose e coma (GANONG, 2010).

A Sociedade Brasileira de Diabetes (2007) tem alertado que, as estimativas para a população brasileira são extremamente preocupantes, pois o país ocupa a sétima posição mundial em relação ao número de diabéticos na faixa etária de 35 a 64 anos e se estima que serão 11,3 milhões de diabéticos em 2030 no país, ou seja, mais que o dobro do número registrado em 2000 (4,5 milhões).

As doenças crônicas não transmissíveis são de alto custo para o Sistema Único de Saúde (SUS), que caso não sejam assistidas de forma adequada, com medidas de tratamento e prevenção, o índice de mortalidade nesse grupo aumentará. Passos et al. (2006), mostram que, fazem parte das estratégias de políticas de promoção a saúde, a detecção dos grupos de risco e a adoção de ações preventivas, partindo dessa problemática. Essas medidas devem diminuir a incidência de doentes crônicos, proporcionando uma melhoria na qualidade de vida.

Diante dessas séries de problemas que norteiam a população idosa, é necessária a inserção de programas preventivos e que acompanhem periodicamente esse grupo, estimulando o abandono dos 
maus hábitos, e a adoção de práticas que visem o bem-estar, na tentativa de assim, manter o controle dessas doenças afim de aumentar a qualidade de vida dos mesmos, pois o idoso doente é resultado de uma vida negligenciada em relação aos cuidados primordiais com a saúde. A pesquisa avaliou os fatores de risco para doenças cardiovasculares na população de terceira idade de Manaus, medindo o peso, altura, circunferências e pressão arterial para as análises.

\section{MATERIAL E MÉTODOS}

A pesquisa sucedeu-se entre o período de agosto de 2014 a julho de 2015, nas seguintes localizações: Fundação Dr. Thomas, Rua Fundação Dr. Thomas, nº 798, Bairro Nossa Senhora das Graças; e Parque Municipal do Idoso, Rua Rio Mar, n 1324, também localizado no Bairro Nossa Senhora das Graças.

Antes da realização da pesquisa, foi entregue aos idosos o Termo de Compromisso Livre e Esclarecido conforme registrado no comitê de ética de $N^{\circ}$ CAAE: 31168114.5 .0000 .5016 , (vinculado a Plataforma Brasil) juntamente com o questionário de perfil familiar e alimentar, no qual se mostrariam solícitos para a participação da pesquisa. Foram ressaltados que, de acordo com os padrões éticos, seus nomes seriam preservados e aproveitados somente de suas informações os dados que interessam a pesquisa, bem como a idade e sexo do voluntário; participaram 123 idosos, sendo 37 dos idosos residentes da Fundação Dr. Thomas e 86 idosos participantes das atividades recreativas oferecidas pelo Parque Municipal do Idoso, representando assim uma amostra da população idosa da cidade de Manaus. A cada visita nas Instituições foi coletado uma amostra de dados de 20 voluntários, no horário acordado conforme a junta administrativa.

A pesquisa se deu em duas fases: a primeira, para a distribuição dos termos, juntamente com avaliação de histórico familiar e alimentar e a coletas dos dados antropométricos; na segunda fase, foi a realização da palestra de retorno, abordando os temas Hipertensão e Diabetes como fator de risco para doenças cardiovasculares, finalizando com a discussão dos problemas que circundam esse grupo. Os idosos que apresentaram situação de risco para cada um dos itens antropométricos foi direcionado ao setor responsável para que devidas providências fossem tomadas.

Para a medição do peso e altura, na realização do calculo do IMC - Índice de Massa Corporal, foi utilizada uma balança digital da marca Balmak Actlife, com capacidade de suportar até 180kg, e utilizada duas fitas métricas que fora cuidadosamente colocada na parede para facilitar o processo. Para o cálculo do Índice de assa Corporal foi utilizada a fórmula IMC= Peso $(\mathrm{Kg}) /(\text { altura }(\mathrm{m}))^{2}$. Todos os dados que foram obtidos na realização desse trabalho foram comparados com as tabelas da OMS Organização Mundial de Saúde e Sociedade Brasileira de Diabetes. 
Na medição da circunferência abdominal, braço e cintura, para obtenção do número de indivíduos com risco para doenças cardiovasculares, foi utilizada uma fita antropométrica. Para análise dos dados foi utilizada as informações oferecidas pela Organização Mundial da Saúde - OMS. A pressão arterial foi aferida com monitor digital semiautomático G-TECH, onde sentou-se o participante com o braço relaxado sobre a mesa, pediu-se para que o paciente pudesse ficar quieto e em silêncio, pois o aparelho era sensível a qualquer movimento, o que poderia causar alteração no resultado. $\mathrm{O}$ mesmo equipamento já fornece a frequência cardíaca.

Com o material descartável, como lancetas Accu Chek, Fitas, aparelho de medida G Tech, foi realizado a medição da Glicemia Pós prandial, pois os idosos já se encontravam no estado alimentado, onde contou com uma técnica em patologia clínica. Após a coleta dos dados foram feitas as análises utilizando computador com programa excel e plotados os dados em gráficos e tabelas comparativos, separados em grupos de acordo com as semelhanças e variáveis para efetuação de análises e comparações.

\section{APRESENTAÇÃO E ANÁLISE DOS RESULTADOS}

\subsection{PERFIL EPIDEMIOLÓGICO E PSICOSSOCIAL DA POPULAÇÃO}

Participaram da pesquisa 123 voluntários dos quais 76 eram mulheres (62\%) e 47 eram homens (38\%). Os participantes de ambas as instituições se encontravam na faixa etária de 60 a 99 anos (Fundação Dr. Thomas, Média=71,02; D.P=23,44; Parque Municipal do Idoso, Média= 70,09; D.P= 7,65), sendo que, 37 são residentes da Fundação Dr. Thomas (57\% mulheres e $43 \%$ homens); e 86 são participantes rotativos do Parque Municipal do Idoso (64\% mulheres e 36\% homens).

A análise dos resultados dos idosos se diferenciou conforme o perfil comportamental de cada grupo de idoso de acordo com a Instituição ao qual pertence. Na Fundação Dr. Thomas, dos 37 idosos residentes $8 \%$ afirmaram ser fumantes e consumir bebidas alcoólicas; enquanto dos idosos participantes do Parque Municipal do Idoso, de 86 voluntários, a taxa de fumantes foi um pouco menor, perfazendo 5\% dos pesquisados, e 9\% afirmaram o consumo de bebidas alcoólicas.

Simonetti et al. (2002) esclarece dizendo que, apesar de não haver uma relação direta entre o fumo e a hipertensão arterial, o que acontece em relação a arteriosclerose, pois o tabaco comprometerá integridade dos vasos sanguíneos, a nicotina libera catecolaminas, aumentando a frequência cardíaca, resistência periférica e consequentemente, a pressão arterial. Logo, nos fumantes, a hipertensão pode se manifestar de forma maligna, acarretando a incidência elevada de morte súbita. Em relação ao alcoolismo, o mesmo destaca que, o aumento da taxa de álcool no sangue, eleva a pressão arterial, lenta e progressivamente. 
Os idosos apresentam grande disposição para a prática de Exercícios físicos. Os participantes da Fundação Dr. Thomas, no seu contexto geral, de acordo com a mobilidade, obedecem três graus de dependência, onde foi possível a realização da pesquisa somente com os que não necessitam de apoio para se locomover e executam ainda suas atividades de forma independente. Estes, em sua totalidade, realizam a prática de exercícios físicos, embora seja para fins terapêuticos, mostram-se bastante ativos. Os idosos participantes do Parque Municipal do Idoso, em sua maioria, realizam a prática de exercícios físicos, perfazendo uma quantidade de $86 \%$ do total, fator importante, pois contribui para a promoção de uma vida saudável isenta de doenças que podem comprometer a saúde física e mental dos idosos.

A prática de exercícios físicos melhora a capacidade funcional e a aptidão física. Capacidade essa que, ajuda na realização das tarefas do cotidiano com vigor e energia, demonstrando assim menor risco de desenvolver doenças crônico degenerativas, associados aos baixos níveis de atividade física (NAHAS,2001).

Em relação aos hábitos alimentares, os idosos da Fundação Dr. Thomas seguem uma dieta equilibrada, conforme a necessidade e particularidade de cada um, sendo assistidos diariamente por uma nutricionista. Já os idosos do Parque do Idoso não possuem esse privilégio, pois os mesmos realizam sua própria rotina alimentar não tendo assim acompanhamento nutricional, podendo comer do que quiserem ou até mesmo ditar a quantidade de refeições que comem por dia, no qual, dos 27 que responderam o questionário, $48 \%$ realizam 3 refeições diárias, 37\% com 4 refeições, e $4 \%$ realizam 6 refeições completas.

Garcia et al. (2007) afirmam que, existe uma relação entre o envelhecimento e o estado nutricional do indivíduo. Alterações ocorrentes no organismo, como a redução do olfato, diminuição de secreções que auxiliam na digestão, dificuldade de mastigação e constipação são fatores que interferem no estado nutricional do idoso. O que são informações relevantes para os que cuidam desses idosos, pois os riscos de mortalidade diminuirão, ajudando os mesmos a se tornarem mais independentes, melhorando a qualidade de vida.

\subsection{ANÁLISE QUALITATIVA E QUANTITATIVA DE MASSA CORPORAL - IMC}

Durante a realização da pesquisa, foi possível através do cálculo do IMC, observar que a maioria dos idosos, tanto da Fundação Dr. Thomas, quanto do Parque Municipal do Idoso, apresentavam-se acima do peso. De acordo com o padrão estabelecido pela OMS- Organização Mundial da Saúde, 44\% apresentaram-se com sobrepeso (De 25 a 29,9) e 19\% eram obesos (n=34). Dos 37 participantes, 8\% não participaram das medições para a determinação do IMC (Tabela 1). 
Tabela 1. Índice de massa corpórea dos Idosos da Fundação Dr. Thomas.

\begin{tabular}{|c|c|c|c|c|c|}
\hline CATEGORIA & $\begin{array}{l}\text { IMC } \\
\text { PADRÃO* }\end{array}$ & PARTICIPANTES & $(\%)^{* *}$ & FEMININO (\%) & $\begin{array}{l}\text { MASCULINO } \\
(\%)\end{array}$ \\
\hline Abaixo do peso & $\begin{array}{ll}\text { Abaixo } & \text { do } \\
18,5 & \end{array}$ & 3 & 8 & 5 & 3 \\
\hline Peso normal & $\begin{array}{l}\operatorname{De} 18,5 \quad \text { a } \\
24,9\end{array}$ & 12 & 32 & 16 & 16 \\
\hline Sobrepeso & De 25 a 29,9 & 11 & 29 & 13 & 16 \\
\hline Obesidade Grau I & $\begin{array}{l}\text { De } 30,0 \quad \text { a } \\
34,9\end{array}$ & 4 & 11 & 11 & 0 \\
\hline Obesidade Grau II & 35,0 a 39,9 & 3 & 8 & 0 & 8 \\
\hline $\begin{array}{l}\text { Obesidade Grau } \\
\text { III }\end{array}$ & $>=40$ & 1 & 3 & 3 & \\
\hline TOTAL DE PARTI & CIPANTES: & 34 & 92 & & \\
\hline
\end{tabular}

* Padrão: OMS - Organização Mundial de Saúde (World Health Organization), 2007

De acordo com Wanderley e Ferreira (2010), a obesidade é uma doença causada pelo acúmulo excessivo de gordura corporal, comprometendo a saúde do indivíduo, devido a alterações metabólicas, causando dificuldades respiratórias, comprometendo o aparelho locomotor, o que contribui para o aparecimento de doenças cardiovasculares, diabetes do tipo I e II, bem como o câncer. Segue abaixo o perfil da população idosa quanto ao IMC, fator de risco para a obesidade. Em relação aos idosos do Parque Municipal do Idoso, 63\% dos idosos apresentaram-se acima do peso ( $n=7)$, dos 86 participantes, $10 \%$ não participaram das medições para o IMC (Tabela 2).

Tabela 2. Índice de massa corpórea dos Idosos do Parque do Idoso

\begin{tabular}{|c|c|c|c|c|c|}
\hline CATEGORIA & $\begin{array}{l}\text { IMC } \\
\text { PADRÃO* }\end{array}$ & PARTICIPANTES & $(\%)^{* *}$ & FEMININO (\%) & $\begin{array}{l}\text { MASCULINO } \\
(\%)\end{array}$ \\
\hline Abaixo do peso & $\begin{array}{ll}\text { Abaixo do } \\
18,5\end{array}$ & 2 & 2 & 2 & 0 \\
\hline Peso normal & $\begin{array}{l}\operatorname{De} 18,5 \quad \text { a } \\
24,9\end{array}$ & 20 & 23 & 11,5 & 11,5 \\
\hline Sobrepeso & De 25 a 29,9 & 38 & 44 & 24 & 20 \\
\hline Obesidade Grau I & $\begin{array}{l}\text { De } 30,0 \text { a } \\
34,9\end{array}$ & 8 & 9 & 8 & 1 \\
\hline Obesidade Grau II & 35,0 a 39,9 & 7 & 8 & 5 & 3 \\
\hline $\begin{array}{l}\text { Obesidade Grau } \\
\text { III }\end{array}$ & $>=40$ & 2 & 2 & 2 & 0 \\
\hline TOTAL DE PARTI & CIPANTES: & 77 & 90 & & \\
\hline
\end{tabular}

* Padrão: OMS - Organização Mundial de Saúde (World Health Organization), 2007

O excesso de peso que cresce em 1/3 da população adulta, tem se repetido na população idosa. Há uma prevalência maior de obesidade entre as mulheres, inclusive nos idosos (4,5\% a mais). Em ambos os sexos, seu maior pico ocorre entre 45 e 64 anos (WHO, 1997). Mas, infelizmente, os índices de sobrepeso e obesidade já são encontrados em adolescentes e universitários, em estudo realizado em 
escolas públicas de Manaus, com porcentagem significativa e preocupante (RIBEIRO; FREITAS; ROSA-OSMAN, 2020).

\subsection{IDENTIFICAÇÃO DOS FATORES DE RISCO PARA DOENÇAS CARDIOVASCULARES}

Na medição da circunferência para os riscos de doenças cardiovasculares, os participantes da Fundação Dr. Thomas apresentaram os seguintes resultados: 24\% apresentaram o risco aumentado e $48 \%$ com o risco muito aumentado $(n=36)$. Dos 37 participantes, $4 \%$ deixaram de medir a circunferência (Tabela 3). Esses dados refletem uma situação muito grave, pois é um fator indicativo de que tanto esse grupo, quanto o grupo do Parque do idosos, os indivíduos são fortes candidatos a sofrerem infartos.

A OMS - Organização Mundial da saúde (2011), classifica as doenças cardiovasculares - DCV, como o conjunto de doenças que afetam o sistema circulatório, representando um grande problema para a saúde pública, por ser um grande indicador de mortalidade no mundo todo.

Trata-se de uma doença multifatorial oriundo de vários fatores como: susceptibilidade genética, idade, presença de hipertensão arterial, diabetes mellitus, dislipidemias, obesidade, inatividade física, hábitos alimentares inadequados, tabagismo, etilismo e estresse. Cada um desses fatores, possui sua particularidade em ao influenciar no aparecimento das doenças cardiovasculares, onde, quanto maior os riscos do indivíduo se expor a esses fatores, maiores serão as chances de desenvolvimento da doença (CARVALHO et al., 2008).

A relação entre fatores de risco e DCV, a incidência de doença coronariana e infarto aumentam com a idade, sendo que mais da metade das pessoas com doenças coronarianas têm pelo menos 65 anos e uma história familiar positiva para cardiopatia (SMELTZER et al.,2009).

Tabela 3: Dados da circunferência para riscos de doenças cardiovasculares: Idosos da Fundação Dr. Thomas.

\begin{tabular}{|c|c|c|c|c|c|}
\hline CATEGORIA & PADRÃO* & PARTICIPANTES & $(\%)^{* *}$ & $\begin{array}{l}\text { FEMININO } \\
(\%)\end{array}$ & $\begin{array}{l}\text { MASCULINO } \\
(\%)\end{array}$ \\
\hline $\begin{array}{l}\text { Circunferência } \\
\text { normal }\end{array}$ & $\begin{array}{l}<80 \text { para } \\
\text { mulheres e }< \\
94 \quad \text { para } \\
\text { homens }\end{array}$ & 9 & 24 & 3 & 21 \\
\hline Risco Aumentado & $\begin{array}{l}>80 \text { para } \\
\text { mulheres e } \\
>94 \text { para } \\
\text { homens }\end{array}$ & 9 & 24 & 16 & 8 \\
\hline $\begin{array}{l}\text { Risco Muito } \\
\text { Aumentado }\end{array}$ & $\begin{array}{l}>88 \text { para } \\
\text { mulheres } \mathrm{e} \\
>102 \text { para } \\
\text { homens }\end{array}$ & 18 & 48 & 35 & 13 \\
\hline TOTAL DE PARTI & IPANTES: & 36 & 96 & & \\
\hline
\end{tabular}

* Padrão: WHO - World Health Organization (Organização Mundial de Saúde) 1997

** Número Percentual de participantes. 
Ao envelhecer, o indivíduo passa por transformações fisiológicas caracterizando o seu perfil antropométrico. Segundo, Sten (1988), essas modificações estão associadas a perda progressiva da massa magra com aumento da proporção de gordura corpórea, além da diminuição da estatura, relaxamento da musculatura abdominal, cifose e alteração da elasticidade da pele.

Para os participantes do Parque Municipal do Idoso, a tabela mostra dados cada vez mais preocupantes, onde $22 \%$ apresentaram o risco aumentado e $67 \%$ com o risco muito aumentado $(n=85)$ (Tabela 4). Para Lima-Costa et al., (2000), as doenças cardiovasculares constituem o principal grupo de causas de mortalidade entre idosos, em países como os Estados Unidos da América e o Brasil. Para Francischi et al., (2000), a gordura abdominal altera o metabolismo levando a riscos cardiovasculares como dislipidemia, hipertensão arterial e diabetes mellitus.

\begin{tabular}{|c|c|c|c|c|c|}
\hline CATEGORIA & PADRÃO* & PARTICIPANTES & $(\%) * *$ & $\begin{array}{l}\text { FEMININO } \\
(\%)\end{array}$ & $\begin{array}{l}\text { MASCULINO } \\
(\%)\end{array}$ \\
\hline $\begin{array}{l}\text { Circunferência } \\
\text { normal }\end{array}$ & $\begin{array}{l}<80 \text { para } \\
\text { mulheres e }< \\
94 \quad \text { para } \\
\text { homens }\end{array}$ & 8 & 9 & 5 & 2 \\
\hline Risco Aumentado & $\begin{array}{l}>80 \text { para } \\
\text { mulheres } \mathrm{e} \\
>94 \text { para } \\
\text { homens }\end{array}$ & 19 & 22 & 6 & 16 \\
\hline $\begin{array}{l}\text { Risco } \\
\text { Aumentado }\end{array}$ & $\begin{array}{l}>88 \text { para } \\
\text { mulheres } \mathrm{e} \\
>102 \text { para } \\
\text { homens }\end{array}$ & 58 & 67 & 50 & 17 \\
\hline
\end{tabular}

TOTAL DE PARTICIPANTES: $\quad 85 \quad 99$

* Padrão: WHO - World Health Organization (Organização Mundial de Saúde) 1997.

**Número Percentual de participantes

\section{4 ÍNDICE DE GLICEMIA CAPILAR}

Dos idosos de cada Instituição, foi selecionado aleatoriamente um grupo para a medição da Glicemia Pós-prandial, para a detecção da quantidade de glicose sanguínea. A medição da glicemia periódica foi importante para a determinação dos indivíduos diabéticos. O diabetes está relacionado ao comprometimento de várias vias metabólicas, e o corpo, para suprir a falta de energia, utiliza das vias de reserva energética, muitas vezes, obrigando o cérebro a utilizar corpos cetônicos, para produção de energia, sendo uma alternativa extremamente tóxica ao organismo, comprometendo assim a homeostase sanguínea e fisiológica, devido a cetoacidose metabólica, que para Esmeltser e Bare (2009), se houver o acumulo dessas substâncias na circulação acarretam graves prejuízos fisiológicos, pois o ph ácido é incompatível com a vida. 
Para o levantamento do índice glicêmico, o resultado em relação aos Idosos residentes da Fundação Dr. Thomas $(\mathrm{n}=10)$ foi que, 5,4\% apresentaram-se diabéticos, 16,2\% dos idosos encontraram-se dentro dos limites e 5,4\% com o Índice bom (Tabela 5).

Tabela 5: Classificação da Glicemia Pós-Prandial dos Idosos da Fundação Dr. Thomas.

\begin{tabular}{llllll}
\hline CATEGORIA & $\begin{array}{l}\text { PADRÃO* } \\
(\mathrm{mg} / \mathrm{dl})\end{array}$ & PARTICIPANTES & $(\%)^{* *}$ & $\begin{array}{l}\text { HOMEM } \\
(\%)\end{array}$ & $\begin{array}{l}\text { MULHER } \\
(\%)\end{array}$ \\
\hline Bom & $70-110$ & 2 & 5,4 & 2,7 & 2,7 \\
Aceitável & $110-140$ & 6 & 16,2 & 2,7 & 13,5 \\
Insatisfatório & $>140$ & 2 & 5,4 & 0 & 5,4 \\
TOTAL DE PARTICIPANTES & 10 & 27 & & \\
\hline
\end{tabular}

*Padrão estabelecido conforme a Sociedade Brasileira de Diabetes (2007).

**Percentual de participantes da pesquisa de acordo com a categoria estabelecida.

A prevalência do diabetes vem crescendo mundialmente, configurando-se atualmente como uma epidemia resultante, em grande parte, do envelhecimento da população. Contudo, o sedentarismo, a alimentação inadequada e o aumento da obesidade também são responsáveis pela expansão global do diabetes. As hospitalizações atribuíveis ao diabetes mellitus representam 9\% dos gastos hospitalares do Sistema Único da Saúde (ROSA e SCHMDIT, 2008).

Para os idosos participantes do Parque do Idoso, $(n=37), 8 \%$ apresentaram-se diabéticos, $21 \%$ encontravam-se dentro dos limites e 14\% com o Índice bom (Tabela 6).

Tabela 6: Classificação da Glicemia Pós-Prandial dos Participantes do Parque Municipal do Idoso.

\begin{tabular}{|c|c|c|c|c|c|}
\hline CATEGORIA & $\begin{array}{l}\text { PADRÃO* } \\
(\mathrm{mg} / \mathrm{dl})\end{array}$ & PARTICIPANTES & $(\%)^{* *}$ & $\begin{array}{l}\text { HOMEM } \\
(\%)\end{array}$ & $\begin{array}{l}\text { MULHER } \\
(\%)\end{array}$ \\
\hline Bom & $70-110$ & 12 & 14 & 3,5 & 10,5 \\
\hline Aceitável & $110-140$ & 18 & 21 & 8 & 13 \\
\hline Insatisfatório & $>140$ & 7 & 8 & 2 & 6 \\
\hline \multicolumn{2}{|c|}{ TOTAL DE PARTICIPANTES } & 37 & 43 & & \\
\hline
\end{tabular}

*Padrão estabelecido conforme a Sociedade Brasileira de Diabetes (2007).

**Percentual de participantes da pesquisa de acordo com a categoria estabelecida.

A insulina é produzida pelo pâncreas. Quando esse hormônio age normalmente, a taxa de glicose diminui e nosso organismo tem a energia necessária para levar uma vida plena e ativa. Nas pessoas com diabetes, esse sistema não funciona bem. Quando se tem diabetes, nosso corpo não pode produzir energia a partir dos alimentos que consome. A glicose pode aumentar no sangue, e não chega até as células do organismo, processo fundamental para uma vida saudável. Então, sem insulina, a glicose consumida (doces, frutas, massas) não consegue ser transformada em energia dentro das células e a pessoa fica com a taxa de glicose no sangue elevada, hiperglicemia (SARTORELLI; FRANCO, 2006). 


\subsection{DADOS DA AFERIÇÃO DA PRESSÃO ARTERIAL SISTÊMICA}

Causada pelo aumento da pressão arterial, a Hipertensão é uma doença perigosa, pois se manifesta de forma silenciosa na população e independe da idade, pois está atrelada a ausência de uma alimentação saudável, rica em sódio e a falta de exercício físico comprometendo o bom funcionamento fisiológico do organismo, sendo que com isso, o indivíduo que sofre de hipertensão poderá desenvolver alguns problemas sistemáticos, pois a pressão alta estimula os órgãos a trabalharem mais, aumentando o volume, acarretando vários problemas fisiológicos.

Em algumas pessoas, a hipertensão mostra-se de forma assintomática, mas em algumas pessoas pode provocar nictúria, cefaleia unilateral, dificuldades de concentração, sudorese, ansiedade e irritabilidade excessivas, edema, fadiga fácil, palpitação e pulso periférico cheio. (MICHELINI, 2000).

A HAS é o mais importante fator de risco cardiovascular modificável, estando associada a condições bastante frequentes em idosos, como doença arterial coronária (DAC), doença cerebrovascular (DCV), insuficiência cardíaca (IC), doença renal terminal, doença vascular periférica, hipertrofia ventricular esquerda (HVE) e disfunção diastólica. Porém foi demonstrado, por vários estudos, que o tratamento anti-hipertensivo reduz o risco dessas complicações catastróficas (MIRANDA et al.,2002).

Dos dados provenientes da pesquisa dos participantes da Fundação Dr. Thomas (n=34) os resultados que chamaram mais atenção para pré-disposição de Hipertensão foi que 13,5\% apresentaram Pressão Normal Alta, 35\% com hipertensão Grau 1 e 8\% com hipertensão Grau 2. Dos 37 idosos, 8\% deixaram de aferir a pressão (Tabela 7). Segundo Miranda et al. (2002) as alterações causadas na aorta, devido ao envelhecimento fisiológico da artéria, exercem um importante papel na gênese da HAS Hipertensão Arterial Sistólica.

Tabela 7: Dados da P.A. dos Idosos da Fundação Dr. Thomas.

\begin{tabular}{|c|c|c|c|c|c|}
\hline CATEGORIA & $\begin{array}{l}\text { Pressão Sistólica e } \\
\text { Diastólica (mmHg) } \\
\text { PADRÃO* }\end{array}$ & PARTICIPANTES & $(\%)^{* *}$ & HOMEM (\%) & $\begin{array}{l}\text { MULHER } \\
(\%)\end{array}$ \\
\hline Ótima & $<120$ e $<80$ & 8 & 22 & 11 & 11 \\
\hline Normal & $120-129$ e $80-84$ & 5 & 13,5 & 5 & 8,5 \\
\hline Normal Alta & $130-139$ e $85-89$ & 5 & 13,5 & 11 & 3,5 \\
\hline Hipertensão 1 & $140-159$ e $90-99$ & 13 & 35 & 27 & 8 \\
\hline Hipertensão 2 & $\begin{array}{l}160-179 \text { e } 100- \\
109\end{array}$ & 3 & 8 & 5 & 3 \\
\hline Hipertensão 3 & $\begin{array}{l}\text { Maior ou igual a } \\
180 \text { e Maior ou } \\
\text { igual a } 110\end{array}$ & 0 & 0 & 0 & 0 \\
\hline \multicolumn{2}{|c|}{ TOTAL DE PARTICIPANTES: } & 34 & 92 & & \\
\hline
\end{tabular}

*Padrão conforme os dados oferecidos pela Organização Mundial da Saúde - OMS.

** Número percentual de participantes 
Os idosos frequentadores do Parque Municipal do Idoso, apesar de possuírem um índice um pouco menor de hipertensos é considerável o número de idosos propensos a adquirir hipertensão, caso não se previnam, modificando comportamentos, principalmente alimentares. Os dados apontam para 28\% com Pressão Normal Alta, 23\% apresentam-se com hipertensão Grau 1 e 12\% com hipertensão Grau 2 (n=83). Dos 86 participantes, 4\% deixaram de aferir a pressão arterial.

Tabela 8. Dados da P.A. Idosos do Parque Municipal do Idoso.

\begin{tabular}{|c|c|c|c|c|c|}
\hline CATEGORIA & $\begin{array}{l}\text { Pressão } \\
\text { Sistólica } \\
\text { Diastólica } \\
\text { (mmHg) } \\
\text { PADRÃ } *\end{array}$ & PARTICIPANTES & $(\%)$ & HOMEM (\%) & $\begin{array}{l}\text { MULHER } \\
(\%)\end{array}$ \\
\hline Ótima & $<120$ e $<80$ & 12 & 14 & 10,5 & 3,5 \\
\hline Normal & $\begin{array}{l}120-129 \text { e } 80- \\
84\end{array}$ & 17 & 18 & 12 & 6 \\
\hline Normal Alta & $\begin{array}{l}130-139 \text { e } 85- \\
89\end{array}$ & 23 & 28 & 9 & 19 \\
\hline Hipertensão 1 & $\begin{array}{l}140-159 \text { e } 90- \\
99\end{array}$ & 20 & 23 & 8 & 15 \\
\hline Hipertensão 2 & $\begin{array}{l}160-179 \text { e } 100 \\
-109\end{array}$ & 10 & 12 & 7 & 5 \\
\hline Hipertensão 3 & $\begin{array}{l}\text { Maior ou igual a } \\
180 \text { e Maior ou } \\
\text { igual a } 110\end{array}$ & 1 & 1 & 1 & \\
\hline \multicolumn{2}{|c|}{ TOTAL DE PARTICIPANTES: } & 83 & 96 & & \\
\hline
\end{tabular}

Costa et al. (2004) afirma que a prevalência da hipertensão nos idosos é superior a 60\%, tornando-se fator determinante na morbimortalidade dessa população, exigindo assim correta identificação do problema e a apropriada abordagem terapêutica. $\mathrm{O}$ controle da hipertensão se faz por meio de tratamento medicamentoso contínuo além de mudanças no estilo de vida, exigindo de seus portadores controle durante toda a vida.

A Organização Pan-Americana da Saúde e Organização Mundial de Saúde (2015), criaram o Dia mundial da Hipertensão em 2006, em todo dia 17 de maio, a fim de dar um retorno dos números de casos de hipertensão em relação a população Mundial, com o intuito realizar uma conscientização. Relataram que, 30\% de a população adulta sofrem de hipertensão, acima de 140/90 mmHg, um terço delas desconhece sua condição. Apesar de ser mais comum em homens, mulheres acima de 65 anos também tem hipertensão.

Preocupado com a situação da população brasileira em relação a hipertensão, especialmente os idosos, que fazem parte do grupo de risco, o Ministério da Saúde implantou o programa Hiperdia, destina-se ao cadastramento e acompanhamento desse grupo de risco, atendidos na rede ambulatorial do Sistema Único de Saúde - SUS, para a realização de todos os procedimentos necessários para 
garantir a saúde dos mesmos. O sistema envia dados para o Cartão Nacional de Saúde, funcionalidade que garante a identificação única do usuário do Sistema Único de Saúde - SUS.

\section{CONSIDERAÇÕES FINAIS}

Levando se em consideração todos os resultados apresentados anteriormente, chega-se à conclusão de que, com o avanço da idade, o indivíduo começa a apresentar diversos problemas que comprometem o funcionamento fisiológico de seu organismo, decorrente na maioria das vezes de uma vida negligenciada as boas práticas de promoção a saúde. As Doenças Crônicas não Transmissíveis DCNT's não tem cura, porém se o indivíduo adotar o hábito de ter uma dieta balanceada com frutas, legumes, fibras, nutrientes essenciais e vitaminas, aliados com a prática de exercícios físicos, contribuirá satisfatoriamente para a controle da doença, melhorando assim a qualidade de vida, bem como a longevidade. Logo, faz-se necessário que se reforcem os programas de prevenção e tratamento dessas doenças, para que se reverta o perfil da população brasileira e quando a terceira idade chegar, o indivíduo possa viver com tranquilidade, de forma saudável o que está intimamente relacionada ao bom funcionamento do sistema cardiovascular. 


\section{REFERÊNCIAS BIBLIOGRÁFICAS}

BRASIL. Ministério da Saúde. Relatório técnico da campanha nacional de detecção de suspeitos de diabetes mellitus. Brasília: Secretaria de Políticas da Saúde, Ministério da Saúde, 2001.

BRASIL. Ministério da Saúde. Secretaria de Atenção à Saúde. Departamento de Atenção Básica.Hipertensão arterial sistêmica para o Sistema Único de Saúde / Ministério da Saúde, Secretaria de Atenção à Saúde, Departamento de Atenção Básica. - Brasília: Ministério da Saúde, 2006

BRASIL. Ministério da Saúde. Secretaria de Atenção à Saúde. Departamento de Ações Programáticas e Estratégicas. Atenção à saúde da pessoa idosa e envelhecimento / Ministério da Saúde, Secretaria de Atenção à Saúde, Departamento de Ações Programáticas e Estratégicas, Área Técnica Saúde do Idoso. Brasília, 2010.

CARVALHO, G.Q; ALFENAS, R.C.G. Índice glicêmico: uma abordagem crítica acerca de sua utilização na prevenção e no tratamento de fatores de risco cardiovasculares. Rev Nutr [Internet]. 2008 [cited 2013 Apr 02];21(5):57787.

COSTA MF; UCHOA E. Projeto Bambuí: maneiras de pensar e agir de idosos hipertensos. Cad Saúde Pública. 2004;20(4):1029-40.

ESMELTSER, S. C; BARE, B, G. Tratado de Enfermagem Médico-Cirúrgico. 8. Ed. Rio de Janeiro: Guanabara Koogan S.A., 2009.

FRANCISCHI RPP; PEREIRA LO; FREITAS CS; KLOPFER M; SANTOS RC; VIEIRA P, et al. Obesidade: atualização sobre etiologia, morbidade e tratamento. Rev Nutr 2000; 13:17-28

GANONG, W. F. Fisiologia Médica. William F. Ganong; tradução Carlos Henrique Cosendey, Denise Costa Rodrigues, Patricia Voeux; revisão técnica: Antônio Nóbrega. 22 ed. Porto Alegre: AMGH, 2010.

GARCIA, A.N.M; ROMANI, S.A.M; LIRA P.I.C. Indicadores antropométricos na avaliação nutricional de idosos: um estudo comparativo. Rev Nutr 2007;20(4):371-8.

GUYTON, ARTHUR C. Fisiologia Humana. Arthut C. Guyton; tradução Charles Alfred Esberard. (Reimpr.). Rio de Janeiro: Guanabara Koogan, 2011.

LEDERER, J. Enciclopédia Moderna de Higiene Alimentar . São Paulo : Manole Dois, 1991.

LIMA-COSTA, M.F.F; GUERRA, H.L; BARRETO, S.M; GUIMARÃES, R.M. Diagnóstico de saúde da população idosa brasileira: Um estudo da mortalidade e das internações hospitalares públicas. Informe Epidemiológico do SUS. V.9, n.1, 2000. p. 23-41.

MANTOVANI, M.F; VILELA, J.C; GIACOMOZZI, L.M. A percepção de doenças crônicas por acadêmicos de enfermagem. Conclusão de Curso de Enfermagem, 2008.

MICHELINI, L.C. Regulação momento a momento da pressão arterial na normotensão e hipertensão. Sociedade Brasileira de Hipertensão, 2000. p. 90. 
NAHAS, M.V. Atividade física, saúde e qualidade de vida: conceitos e sugestões para um estilo de vida ativo. Londrina: Midiograf, 2001.

OMS. Organização Mundial da Saúde. Nota Informativa: Enfermidades cardiovasculares, set. 2011 [Internet]. Disponível no site: http://www.who.int/mediacentre/factsheets /fs317/es.

PASSOS, V.M; BARRETO, S.M; DINIZ, L.M; LIMA-COSTA, M.F. Type 2 diabetes: prevalence and associated factors in a Brazilian community-the Bambui health and aging study. Sao Paulo Med J. V.123, 2005. n2, p.66-71

PASSOS, V.M; ASSIS, T.D; BARRETO, S.M. Hipertensão arterial no Brasil: estimativa de prevalência a partir de estudos de base populacional. Epidemiol Serv Saude. V15, n.1, 2006. p. 3545.

RIBEIRO, S.C.; FREITAS, J.B.; ROSA-OSMAN, S.M. Avaliação de fatores de risco para doenças cardiovasculares: Estudo com população de estudantes da rede pública de Manaus-AM. Latin American Journal of Development, Curitiba, v.2, p.363-378, 2020. DOI: 10.46814/lajdv2n6-004

ROSA, R.S; SCHMIDT, M.I. Diabetes Mellitus: magnitude das hospitalizações na rede pública do Brasil. 1999-2001. EpidemiolServSaude. ;V.17, n.2,2008. p.131-4.

SARTORELLI, S.D; FRANCO,J.L; C.A.M. Intervenção nutricional e prevenção primária do diabetes mellitus tipo 2: Uma revisão sistemática. Cad. Saúde Pública, Rio de Janeiro, V. 22, n.1, 2006. p. 7-18.

SIDNEY, S; STERNFELD, B; HASKELL, W.L. Seven-year change in graded exercise treadmill test performance in young adults in the CARDIA-study. Med Sci Sports Exerc. 1998;30(3):42733.

SIMONETTI JP.; BATISTA L.; CARVALHO LR. Hábitos de saúde e fatores derisco em pacientes hipertensos. Rev Latino-Am Enfermagem, v. 10(3): p. 1-9, 2002.

SOCIEDADE BRASILEIRA DE DIABETES. Alvo no controle metabólico de crianças e adolescentes com Diabetes mellitus tipo 1. 2007.

SMELTZER, S. C; BARE, B.G. Tratado de Enfermagem Médico-Cirúrgica. In: Histórico e tratamento de Pacientes com Hipertensão. 10 ed. Rio de Janeiro: Guanabara Koogan, 2009.

STEEN B. Body composition and aging. Nut Rev, V.26. n. 2, p.18-23. 1988.

TELEMA, R; YANG, X; LAAKSO, L. \& VIIKAR I, J. Phisical activity in childhood and adolescence of physical activity in young adulthood. Am. J. Prev. Méd., v.13, n.4, p. 317-323, 1997.

WANDERLEY, E. N; FERREIRA, V. A. Obesidade: uma perspectiva plural. Ciência e Saúde Coletiva, 2010.

WHO. Consultation on Obesity: Prevention and Managing: The Global Epidemic. Report of a WHO Consultation on Obesity. Geneva, 1997. 
WRIGHT Jr, J.T; FINE, L.J; LACKAND, D.T; OGEDEDBDE, O; HIMMEFARB, CRD. Evidence Supporting a Systolic Blood Pressure Goal of Less Than $150 \mathrm{~mm} \mathrm{Hg}$ in Patients Aged 60 Years or Older: The Minority View. Ann Intern Med. V.160(7), 2014. p. 499-503. doi: 10.7326/M13-2981. 